\title{
Relations and Panpsychism
}

\author{
Carlo Rovelli \\ AMU Université, Université de Toulon, CNRS, CPT, F-13288 Marseille, EU \\ Perimeter Institute, 31 Caroline Street N, Waterloo ON, N2L2Y5, Canada and \\ The Rotman Institute of Philosophy, 1151 Richmond St. N London N6A5B7, Canada
}

(Dated: September 30, 2021)

\begin{abstract}
20th century physics has revealed a pervasive relational aspect of the physical world. This fact is relevant in view of some of the motivations for panpsychism. In facts, it may be seen as a vindication of the panpsychist idea of a monist continuity where some aspects of the consciousness' perspectivalism are universal. But this same fact undermines the motivations for genuine forms of panpsychism.
\end{abstract}

If some aspects of mind are universal, which ones are so? I point out in this note that 20th Century physics has already vindicated a -very mild-form of panpsychism. This is because of the profoundly relational aspect this physics, manifest in general relativity, but especially in quantum mechanics. 20thCentury physics is not about how individual entities are by themselves. It is about how entities manifest themselves to one another. It is about relations.

This is particularly evident for quantum theory, especially (but not uniquely) if one reads it in terms of its relational interpretation $[1,2]$. Niels Bohr expressed this 'contextual' aspect of quantum theory by saying that a physical system can only be described taking into account the systems it is interacting with. The current account of the world in fundamental physics is therefore always the account of how a system affects another system.

This implies that the most effective way of thinking about the world is not in terms of entities with properties, but rather in terms of systems that have properties in relation to other systems. In turn, this implies that any physical description of a system is necessarily perspectival: relative to another system.

In the textbook Copenhagen formulation of quantum theory, the physical system with respect to which properties take value is variously and a bit obscurely interpreted as 'the apparatus', 'the observer', 'the macroscopic world' and similar. In relational quantum mechanics, the properties of a system $S$ are defined with respect to any other physical system $O$ with which $S$ interacts, and are relative to $O$ [2]. Similarly, in the many-worlds interpretation of quantum mechanics, variables have values only in relation to (components in a branch of) other systems when the two have got entangled [3].

A direct consequence is that our physics today is not a physics of the world seen from the outside. It is a physics or the world always seen from the perspective a physical system.

This is perhaps not panpsychism, because there is nothing specifically psychic or mental in the relational properties of a system with respect to another system. But there is definitely something in common with panpsychism, because the world is not described from the outside: it is always described relative to a physical system [4]. So, physical reality is, in our current physics, perspectival reality [5].

On the other hand, this very relationalism may suffice to resolve the very problems that motivated panpsychism in the first place: do we really need elementary physics to include more aspects in common with the mental world than this? Which ones? All phenomena of which I am aware that are more related to psyche or mind are connected to a brain, a neural system, sensory organs, feed back loops or the like. I cannot imagine anything even vaguely more like psyche or mind, without some structure like those.

A motivation for panpsychism is the idea that there is a 'hard problem' of consciousness [6]. This is the expectation that even if we had figured out how our body works in terms of our current science, there would still be something mysterious about 'consciousness'. This expectation is based upon an intuition: that subjective experience, the 'first person perspective', must be incompatible with the world as we describe it now in physics. For instance, Chalmers argues that it is conceivable to imagine a body with the same physics as my body, but with no subjective experience; hence subjective experience must be over and above this physics [6].

I do not know how Chalmers can 'conceive' this: I can't. The point is that what we can 'conceive' depends on the conceptual structure we have, and this keeps changing and includes a big deal of presuppositions, sometimes wrong. History of science should warn us that trusting 'intuition' blindly can freeze us into wrong ideas. We do not yet fully understand the physics of thunderstorms, but trusting the compelling 'intuition' that lightning and thunder are evidence for the rage of Odin may not be very wise.

More specifically, the intuition of a tension between mental and physical is based on two assumptions. The first is that the subject of experience is an irreducible entity. The second is that the material world is formed by substances having properties. There is indeed a tension between these two concepts that appear radically distinct. In particular, mental phenomena are intentional: they are relative to something else. Material phenomena at first may seem incompatible with this.

Both these assumptions seems to me to be wrong. There is a vast philosophical literature about the first and I will not go into it. Rather, I focus here on the 
second. The intuition of the 'material world' as formed by substance with properties, for instance just particles moving in space, is based on 18th century physics. Today we have a much more subtle view of the physical world. In particular, as I mentioned above, physics today is about relations between systems, about how systems affect one another. In a precise sense, quantum mechanics, in particular, has undermined the idea of an elementary level where components matter can be described independently from anything else.

If our basic understanding of the physical world is in terms of more or less complex systems that interact with one another and affect one another, the discrepancy between the mental ad the physical seems much less dramatic, and I do not see the need for stronger versions of panpsychism.

This obviously is not a solution to the 'easy problem' of consciousness - figuring out the physics of the body when mental processes happen. But it definitely under- cuts, seems to me, the intuition underpinning the belief that there is any 'hard' problem of consciousness. Mental phenomena are like other complex phenomena, and the existence of a subjective perspective is precisely the generic situation in physics: how systems 'appear to one another'.

So, relationalism can be seen as a very mild form of panpsychism. That is, there is something in common between mind and matter: they are ways reality of physical systems manifest itself to other physical systems, which is precisely what our physics currently describes.

The world as we know it today in physics is neither the material world of 18th century mechanic philosophy, neither embodied with any peculiar proto-consciousness. It is a world where physical systems - simple and complexmanifest themselves to other systems -systems and complex - in a way that our physics describes. I see no reason to belief that this should not be sufficient to account for stones, thunderstorms and thoughts.
[1] Carlo Rovelli, 'Relational quantum mechanics,' International Journal of Theoretical Physics 35, 1637-1678 (1996), arXiv:9609002 [quant-ph].

[2] Carlo Laudisa, Federico and Rovelli, 'Relational Quantum Mechanics,' The Stanford Encyclopedia of Philosophy (2021).

[3] S. Saunders, J. Barrett, A. Kent, and D. Wallace, Many Worlds?: Everett, quantum theory, and realism (Oxford
University Press, 2010).

[4] Mauro Dorato, 'Rovelli' s relational quantum mechanics, monism and quantum becoming,' in The Metaphysics of Relations, edited by A Marmodoro and A Yates (Oxford University Press, 2016) pp. 290-324, arXiv:1309.0132.

[5] Carlo Rovelli, Helgoland (Penguin, London, 2021).

[6] David Chalmers, 'Facing Up to the Problem of Consciousness,' Journal of Consciousness Studies 2, 200-219 (1995). 